\title{
New Modification of Behl's Method Free from Second Derivative with an Optimal Order of Convergence
}

\author{
Wartono*, Revia Agustiwari, and Rahmawati \\ Program Studi Matematika, UIN Sultan Syarif Kasim Riau \\ Jalan H.R Subrantas km 15, Simpang Baru, Pekanbaru, Indonesia, 28294 \\ *E-mail: wartono@,uin-suska.ac.id
}

\begin{abstract}
Behl's method is one of the iterative methods to solve a nonlinear equation that converges cubically. In this paper, we modified the iterative method with real parameter $\beta \in \mathfrak{R}$ using second Taylor's series expansion and reduce the second derivative of the proposed method using the equality of Chun-Kim and Newton Steffensen. The result showed that the proposed method has a fourth-order convergence for $\beta=0$ and involves three evaluation functions per iteration with the efficiency index equal to $4^{1 / 3} \approx$ 1.5874. Numerical simulation is presented for several functions to demonstrate the performance of the new method. The final results show that the proposed method has better performance as compared to some other iterative methods.

Keywords: efficiency index; third-order iterative method; Chun-Kim's method; Newton-Steffensen's method; nonlinear equation.
\end{abstract}

\begin{abstract}
Abstrak
Metode Behl adalah salah satu metode iterasi yang digunakan untuk menyelesaikan persamaan nonlinear dengan orde konvergensi tiga. Pada artikel ini, modifikasi terhadap metode iterasi menggunakan ekspansi deret Taylor orde dua dengan parameter $\beta \in \mathfrak{R}$ dan turunan kedua dihilangkan menggunakan penyetaraan dari metode Chun-Kim dan Newton-Steffensen. Hasil kajian menunjukkan bahwa metode iterasi yang diusulkan memiliki orde konvergensi empat untuk $\beta=0$ dan melibatkan tiga evaluasi fungsi setiap iterasinya dengan indeks efisiensi sebesar $4^{1 / 3} \approx 1,5874$. Simulasi numerik dilakukan terhadap beberapa fungsi untuk menunjukkan performa modifikasi metode iterasi yang diusulkan. Hasil akhir menunjukkan bahwa metode iterasi tersebut mempunyai performa lebih baik dibandingkan dengan beberapa metode iterasi lainnya.

Kata kunci: indeks efisiensi; metode iterasi orde tiga; metode Chun-Kim; metode Newton- Steffensen; persamaan nonlinear.
\end{abstract}

\section{INTRODUCTION}

Mathematical modeling is a mathematical representation of sciences and engineering problems. One of the terms of mathematical representation is a nonlinear equation. While in fact, most of the 
nonlinear equation consists of complexity form of function [1]. The problem arises when we solve this equation:

$$
f(x)=0
$$

Most of the complicated nonlinear equations can't be solved using the analytical technique. So, numerical solving is an alternative solution using requiring calculating. This technique is most well known as an iterative method.

The iterative method that are popular to find the root of (1) is the Newton Method i.e.

$$
x_{n+1}=x_{n}-\frac{f\left(x_{n}\right)}{f^{\prime}\left(x_{n}\right)}, n=0,1,2, \ldots
$$

Equation (2) is a one-point iterative method that is constructed by Taylor expansion in first order with quadratic order of convergence. This method involved two evaluation functions with efficiency index is $2^{1 / 2} \approx 1.4142$.

Furthermore, to fix up the order of convergence, second-order Taylor expansion is used to construct an iterative method. It processes appear some one-point iterative method with third-order of convergence, such as Halley's, Halley's Irrational, and Chebyshev's methods [2]. Besides that, some authors develop one-point iterative method with third-order of convergence using several technique approximation, such as quadratic function [3] [4], hyperbolic function [5], parabolic function [6], Adomian decomposition method [7] [8], homotopy perturbation method [7], curvature [9], variation iteration method [10], and substituted Taylor series [11] [12]. All of the third-order iterative methods involve three functional evaluations with index efficiency equal to $3^{1 / 3} \approx 1.4422$.

The one-point iterative methods above have third-order of convergence. So, based on KungTraub conjecture [13], the iterative methods aren't an optimal order of convergence. To improve the convergence order of an iterative method, some author uses several approximations. One of the approaches used is the substitution of the Taylor second-order series as has been done by Chun and Kim [9], Wartono and Nanda [12], and Baghat [11].

In this paper, we construct a new iterative method using a combination of a one-point iterative method developed by Behl et al. [14] and second-order Taylor series. Furthermore, at the end of this section, we give numerical simulation to examine the performance of our proposed method and some iterative methods. The performance will be compared based on the number of iterations, computational of the order of convergence, the absolute value of a function, absolute error and relative error.

\section{METHOD}

In this section, we use some definitions and theorems that are used to construct an iterative method, finding for the order of convergence both of using Taylor series or computational order of convergence, and numerical simulation.

Theorem 1. [15] Let $f$ has $(n+1)$ continuous derivative on $[a, b]$ for some $n \geq 0$, and let $x, x_{0} \in$ $[\mathrm{a}, \mathrm{b}]$. Then there exists a point $\xi$ between $x_{0}$ and $x$ such that

$$
f(x)=f\left(x_{0}\right)+f^{\prime}\left(x_{0}\right)\left(x-x_{0}\right)+\frac{f^{\prime \prime}\left(x_{0}\right)}{2 !}\left(x-x_{0}\right)^{2}+\cdots+\frac{f^{(n)}\left(x_{0}\right)}{n !}\left(x-x_{0}\right)^{n}+R_{n}(x),
$$


where

$$
R_{n}(x)=\frac{f^{(n+1)}(\xi)}{(n+1) !}\left(x-x_{0}\right)^{n+1} \text {. }
$$

Definition 1. [1] Let $f(x)$ be a real function with simple root $\alpha$ and let $\left\{x_{n}\right\}_{n \in N}$ be a sequence of real numbers that converges to $\alpha$. We say that the order of convergence of the sequence is $c$ if there exists a number $p \in \mathbb{R}^{+}$such that:

$$
\lim _{n \rightarrow \infty} \frac{\left|x_{n+1}-\alpha\right|}{\left|\left(x_{n}-\alpha\right)\right|^{p}}=c .
$$

When $c \neq 0$ it is known as the asymptotic error constant. If $p=1,2$ or 3 , the sequence $\left\{x_{n}\right\}$ is said to have linear, quadratic, and cubic convergence, respectively.

Definition 2. [1] Let $e_{n}=x_{n}-\alpha$ be the error in the $n^{\text {th }}$ and let

$$
e_{n+1}=c e_{n}^{p}+\mathrm{O}\left(e_{n}^{p+1}\right) \text {. }
$$

The relation in Equation (3) called as an error equation in the $(n+1)^{\text {th }}$.

Definition 3. [1] Let $d$ be the number of any evaluation of a function or one of its derivatives. The efficiency of the method is measured by the concept of efficiency index and is defined by

$$
I E=p^{1 / d},
$$

where $p$ is the convergence order of the method.

Definition 4. [1] Suppose that $x_{n-1}, x_{n}$ and $x_{n+1}$ are three successive closer to the root $\alpha$. Then the computational order of convergence $\rho$ is approximated by

$$
\rho \approx \frac{\ln \left|\left(x_{n+1}-\alpha\right) /\left(x_{n}-\alpha\right)\right|}{\ln \left|\left(x_{n}-\alpha\right) /\left(x_{n-1}-\alpha\right)\right|} .
$$

\section{RESULT AND DISCUSSION}

\subsection{The Developed Iterative Method}

In this sub-section, the author constructs an iterative method by considering a one-point iterative method developed by Behl et al. [14] and add one real parameter $\beta$ as follows:

$$
x_{n+1}=x_{n}-\frac{8 f\left(x_{n}\right) f^{\prime}\left(x_{n}\right)^{3}}{8 f^{\prime}\left(x_{n}\right)^{4}-\beta\left(4 f^{\prime}\left(x_{n}\right)^{2}+f\left(x_{n}\right) f^{\prime \prime}\left(x_{n}\right)\right) f\left(x_{n}\right) f^{\prime \prime}\left(x_{n}\right)} .
$$


Equation (4) is a third-order iterative method with three functional evaluations and an efficiency index as 1.4422. Furthermore, to modify (4), we consider a second-order Taylor series expansion about $x_{n}$ written as,

$$
f(x)=f\left(x_{n}\right)+\left(x-x_{n}\right) f^{\prime}\left(x_{n}\right)+\frac{\left(x-x_{n}\right)^{2}}{2 !} f^{\prime \prime}\left(x_{n}\right) .
$$

Let $x_{n+1}$ is a value close to $\alpha$ then $f\left(x_{\mathrm{n}+1}\right) \approx 0$. So, for $x=x_{n+1}$, we can write (5) as:

$$
x_{n+1}-x_{n}=-\frac{f\left(x_{n}\right)}{\frac{\left(x_{n+1}-x_{n}\right)}{2} f^{\prime \prime}\left(x_{n}\right)+f^{\prime}\left(x_{n}\right)} .
$$

The term $x_{n+1}-x_{n}$ at the right of (6) will be substituted by (4). Now, we can rewrite (6) as follows

$$
x_{n+1}-x_{n}=-\frac{f\left(x_{n}\right)}{f^{\prime}\left(x_{n}\right)}\left(\frac{f^{\prime}\left(x_{n}\right)^{2}-\left(\left(8 f^{\prime}\left(x_{n}\right)^{2}+4 \beta f\left(x_{n}\right) f^{\prime \prime}\left(x_{n}\right)\right)+\beta f\left(x_{n}\right)^{2} f^{\prime \prime}\left(x_{n}\right)^{2}\right)}{-8 f^{\prime}\left(x_{n}\right)^{4}+f\left(x_{n}\right) f^{\prime \prime}\left(x_{n}\right)\left(\beta f\left(x_{n}\right) f^{\prime \prime}\left(x_{n}\right)+4 \beta f^{\prime}\left(x_{n}\right)^{2}+4 f^{\prime}\left(x_{n}\right)^{2}\right)}\right)
$$

To fix up the order of convergence and avoid using the second derivative of $f(x)$, we approach $f^{\prime \prime}\left(x_{n}\right)$ using the equality of two third-order iterative methods. We consider Chun-Kim's [9] and Newton-Steffensen's methods [4], respectively, written as follows:

$$
x_{n+1}=\frac{f\left(x_{n}\right)^{2} f^{\prime \prime}\left(x_{n}\right)+2 f\left(x_{n}\right) f^{\prime}\left(x_{n}\right)^{2}}{2 f^{\prime}\left(x_{n}\right)^{3}},
$$

and

$$
x_{n+1}=\frac{f\left(x_{n}\right)^{2}}{f^{\prime}\left(x_{n}\right)\left(f\left(x_{n}\right)-f\left(y_{n}\right)\right)}
$$

where $y_{n}=x_{n}-\frac{f\left(x_{n}\right)}{f^{\prime}\left(x_{n}\right)}$.

Both of iterative method of (8) or (9) have third order of convergence. Based on these equations, $f^{\prime \prime}(x)$ will be approximated using equality of (8) and (9) as Wartono et al. [16] which is written as

$$
\frac{f\left(x_{n}\right)^{2} f^{\prime \prime}\left(x_{n}\right)+2 f\left(x_{n}\right) f^{\prime}\left(x_{n}\right)^{2}}{2 f^{\prime}\left(x_{n}\right)^{3}}=\frac{f\left(x_{n}\right)^{2}}{f^{\prime}\left(x_{n}\right)\left(f\left(x_{n}\right)-f\left(y_{n}\right)\right)},
$$

Using simplification, we obtain a form of $f^{\prime \prime}\left(x_{n}\right)$ as follows

$$
f^{\prime \prime}\left(x_{n}\right)=\frac{2 f^{\prime}\left(x_{n}\right)^{2} f\left(y_{n}\right)}{f\left(x_{n}\right)\left(f\left(x_{n}\right)-f\left(y_{n}\right)\right)},
$$

Furthermore, by substituting (10) into (7) we obtain an equation to iterative method 
New Modification of Behl's Method Free from Second Derivative with an Optimal Order of...

$$
x_{n+1}=x_{n}-\left(\frac{f\left(x_{n}\right)}{f^{\prime}\left(x_{n}\right)}\right)\left(\frac{-2 f\left(x_{n}\right)^{2}+(2 \beta+4) f\left(y_{n}\right) f\left(x_{n}\right)+(-\beta-2) f\left(y_{n}\right)^{2}}{-2 f\left(x_{n}\right)^{2}+(2 \beta+6) f\left(y_{n}\right) f\left(x_{n}\right)+(-\beta-4) f\left(y_{n}\right)^{2}}\right)
$$

Equation (11) is a two-point iterative method involving two functions and one of its first derivative.

\subsection{Convergence Analysis}

The theorem 2 describes the order of convergence in (11) using the Taylor series expansion.

Theorem 2. Let $f(x)$ be a real-valued function. Assume that $f(x)$ has first, second and third derivatives in the interval $D$. If $f(x)$ has a simple root $\alpha \in D$ and $x_{0}$ is sufficiently close to $\alpha$, then the iterative method in (11) has fourth-order of convergence for $\beta=0$ and satisfies the following error equation:

$$
e_{n+1}=\left(-c_{2} c_{3}+c_{2}^{3}\right) e_{n}^{4}+O\left(e_{n}^{5}\right)
$$

\section{Proof :}

Let $\alpha$ be a simple root of $f(x)$. Then $f(\alpha)=0$ and $f^{\prime \prime}(\alpha) \neq 0$. Assume that $x_{n}=\alpha+e_{n}$ then expand $f\left(x_{n}\right)$ using the Taylor series about $\alpha$. We have

$$
f\left(x_{n}\right)=f(\alpha)+f^{\prime}(\alpha)\left(x_{n}-\alpha\right)+\frac{f^{\prime \prime}(\alpha)}{2 !}\left(x_{n}-\alpha\right)^{2}+\frac{f^{\prime \prime \prime}(\alpha)}{3 !}\left(x_{n}-\alpha\right)^{3}+\cdots
$$

So, using $x_{n}=\alpha+e_{n}$, Equation (12) can be written as

$$
f\left(x_{n}\right)=f^{\prime}(\alpha)\left(e_{n}+\frac{f^{\prime \prime}(\alpha)}{2 ! f^{\prime}(\alpha)} e_{n}^{2}+\frac{f^{\prime \prime \prime}(\alpha)}{3 ! f^{\prime}(\alpha)} e_{n}^{3}+\frac{f^{(4)}(\alpha)}{4 ! f^{\prime}(\alpha)} e_{n}^{4}+\frac{1}{f^{\prime}(\alpha)} \mathrm{O}\left(e_{n}^{4}\right)\right)
$$

or

$$
f^{\prime}\left(x_{n}\right)=f^{\prime}(\alpha)\left(1+2 c_{2} e_{n}+3 c_{3} e_{n}^{2}+4 c_{4} e_{n}^{3}+5 c_{5} e_{n}^{4}+O\left(e_{n}^{5}\right)\right)
$$

where $c_{j}=\frac{1}{j !} \frac{f^{(j)}(\alpha)}{f^{\prime}(\alpha)}, j=2,3, \ldots$ Based on (13) and (14), we have

$$
\frac{f\left(x_{n}\right)}{f^{\prime}\left(x_{n}\right)}=e_{n}-c_{2} e_{n}^{2}+\left(2 c_{2}^{2}-2 c_{3}\right) e_{n}^{3}+O\left(e_{n}^{4}\right)
$$

Furthermore, using (15) and $x_{n}=e_{n}+\alpha$, we can write (11) as

$$
y_{n}=\alpha+c_{2} e_{n}^{2}-\left(2 c_{2}^{2}-2 c_{3}\right) e_{n}^{3}+O\left(e_{n}^{4}\right) \text {. }
$$

To simplify (16), we write it as $y_{n}=\alpha+s_{n}$, where

$$
s_{n}=c_{2} e_{n}^{2}-\left(2 c_{2}^{2}-2 c_{3}\right) e_{n}^{3}+O\left(e_{n}^{4}\right)
$$

Using Taylor series, the expanding of $f\left(y_{n}\right)$ about $\alpha$ is 


$$
f\left(y_{n}\right)=f(\alpha)+\left(y_{n}-\alpha\right) f^{\prime}(\alpha)+\frac{\left(y_{n}-\alpha\right)^{2}}{2 !} f^{\prime \prime}(\alpha)+\left(y_{n}-\alpha\right)^{3} f^{\prime \prime \prime}(\alpha)+\cdots .
$$

Using $f(\alpha)=0$ and $y_{n}=\alpha+s_{n}$, Equation (18) become

$$
f\left(y_{n}\right)=f^{\prime}(\alpha)\left(s_{n}+c_{2} s_{n}^{2}+c_{3} s_{n}^{3}+c_{4} s_{n}^{4}+\cdots\right) .
$$

Substituted (17) into (19), hence we obtain $f\left(y_{n}\right)$ in form

$$
f\left(y_{n}\right)=f^{\prime}(\alpha)\left(c_{2} e_{n}^{2}+\left(2 c_{2}^{2}-2 c_{3}\right) e_{n}^{3}+\left(5 c_{2}^{3}-7 c_{2} c_{3}+3 c_{4}\right) e_{n}^{4}+O\left(e_{n}^{5}\right) .\right.
$$

From (13) and (20), we obtain the multiple of $f\left(x_{n}\right)$ and $f\left(y_{n}\right)$ as follows

$$
f\left(x_{n}\right) f\left(y_{n}\right)=f^{\prime}(\alpha)^{2}\left(c_{2} \theta e_{n}^{3}+\left(-\theta c_{2}^{2}+\theta 2 c_{3}\right) e_{n}^{4}+O\left(e_{n}^{5}\right),\right.
$$

and the quadratic of $f\left(x_{n}\right)$ and $f\left(y_{n}\right)$, respectively, are

$$
f\left(x_{n}\right)^{2}=f^{\prime}(\alpha)^{2}\left[e_{n}^{2}+2 c_{2} e_{n}^{3}+\left(2 c_{3}+c_{2}^{2}\right) e_{n}^{4}+O\left(e_{n}^{5}\right)\right],
$$

and

$$
f\left(y_{n}\right)^{2}=f^{\prime}(\alpha)^{2}\left[c_{2}^{2} e_{n}^{4}+O\left(e_{n}^{5}\right)\right] .
$$

Also, using (21), (22) and (23), we have

$$
\begin{gathered}
-2 f\left(x_{n}\right)^{2}=f^{\prime}(\alpha)^{2}\left(-2 e_{n}^{2}-c_{2} e_{n}^{3}+\left(-4 c_{3}-2 c_{2}^{2}\right) e_{n}^{4}+O\left(e_{n}^{5}\right)\right), \\
(2 \beta+4) f\left(x_{n}\right) f\left(y_{n}\right)=f(\alpha)^{2}\left((2 \beta+4) c_{2} e_{n}^{3}+\left(-(2 \beta+4) c_{2}^{2}+(8+4 \beta) c_{3}\right) e_{n}^{4}+O\left(e_{n}^{5}\right)\right), \\
(2 \beta+6) f\left(x_{n}\right) f\left(y_{n}\right)=f(\alpha)^{2}\left((2 \beta+6) c_{2} e_{n}^{3}+\left(-(2 \beta+6) c_{2}^{2}+(12+4 \beta) c_{3}\right) e_{n}^{4}+O\left(e_{n}^{5}\right)\right), \\
(-\beta-2) f\left(y_{n}\right)^{2}=f^{\prime}(\alpha)^{2}\left(-(\beta+2) c_{2}^{2} e_{n}^{4}+O\left(e_{n}^{5}\right)\right),
\end{gathered}
$$

and

$$
(-\beta-4) f\left(y_{n}\right)^{2}=f^{\prime}(\alpha)^{2}\left(-(\beta+4) c_{2}^{2} e_{n}^{4}+O\left(e_{n}^{5}\right)\right) .
$$

From (24), (25), (26), (27) and (28), we have

$$
\begin{aligned}
-2 f\left(x_{n}\right)^{2} & +(2 \beta+4) f\left(y_{n}\right) f\left(x_{n}\right)+(-\beta-2) f\left(y_{n}\right)^{2} \\
& =f(\alpha)^{2}\left(-2 e_{n}^{2}+2 c_{2} \beta e_{n}^{3}+\left((1+4 \beta) c_{3}-(8+3 \beta) c_{2}^{2}\right) e_{n}^{4}+O\left(e_{n}^{5}\right)\right),
\end{aligned}
$$

and

$$
\begin{aligned}
-2 f\left(x_{n}\right)^{2} & +(2 \beta+6) f\left(y_{n}\right) f\left(x_{n}\right)+(-\beta-4) f\left(y_{n}\right)^{2} \\
& =f(\alpha)^{2}\left(-2 e_{n}^{2}+2(1+\beta) c_{2} e_{n}^{3}+\left((8+4 \beta) c_{3}-(12+3 \beta) c_{2}^{2}+\right) e_{n}^{4}+O\left(e_{n}^{5}\right)\right) .
\end{aligned}
$$


New Modification of Behl's Method Free from Second Derivative with an Optimal Order of...

Applying (15), (29), and (30) in (11), and then simplify it, we obtain

$$
\begin{aligned}
\left(\frac{f\left(x_{n}\right)}{f^{\prime}\left(x_{n}\right)}\right) & \left(\frac{-2 f\left(x_{n}\right)^{2}+(2 \beta+4) f\left(y_{n}\right) f\left(x_{n}\right)+(-\beta-2) f\left(y_{n}\right)^{2}}{-2 f\left(x_{n}\right)^{2}+(2 \beta+6) f\left(y_{n}\right) f\left(x_{n}\right)+(-\beta-4) f\left(y_{n}\right)^{2}}\right) \\
& =e_{n}+c_{2}^{2} \beta e_{n}^{3}+\left(\left(c_{2} c_{3}(4 \beta+1)+c_{2}^{3}\left(\beta^{2}-\frac{5}{2} \beta-1\right)\right) e_{n}^{4}+O\left(e_{n}^{5}\right) .\right.
\end{aligned}
$$

Substitute (31) into (11), and using $x_{n+1}=e_{n+1}+\alpha$ and $x_{n}=e_{n}+\alpha$, we get a convergence order of the proposed method in (11) that is given by

$$
e_{n+1}=-c_{2}^{2} \beta e_{n}^{3}+\left(-(4 \beta+1) c_{2} c_{3}+\left(-\beta^{2}+\frac{5}{2} \beta+1\right) c_{2}^{3}\right) e_{n}^{4}+O\left(e_{n}^{5}\right)
$$

Equation (32) gives us information that the order of convergence of the proposed method in (11) will increase by taking $\beta=0$. So, Equation (32) can be written as

$$
e_{n+1}=\left(-c_{2} c_{3}+c_{2}^{3}\right) e_{n}^{4}+O\left(e_{n}^{5}\right)
$$

\subsection{Numerical Simulation}

In this section, we present a numerical simulation to show the performance of the proposed method for $\beta=0$ (MMIOT), and then we compare the proposed method between some kind of iterative methods, namely: Newton's method (MN), third-order iterative method (MIOT), NewtonSteffensen's (MNS) [4] and Chun-Kim's method (MCK) [8]. Some measures of performance of the compared methods are the number of iterations (IT), computational order of convergence (COC), and the absolute value of a function at $n^{\text {th }}$ iteration $\left(\left|f\left(x_{n}\right)\right|\right)$.

We used several following real test functions and all computations have been performed using MAPLE 13 with 800 digits floating-point arithmetic. The root of real test functions is displayed by the computed approximate zeros $a$ round up to 20th decimal places.

$$
\begin{aligned}
& f_{1}(x)=x e^{-x}-0.1, \alpha \approx 0.11183255915896296483 \\
& f_{2}(x)=e^{x}-4 x^{2}, \alpha \approx 4.30658472822069929833 \\
& f_{3}(x)=\cos (x)-x, \alpha \approx 0.73908513321516064165 \\
& f_{4}(x)=(x-1)^{3}-1, \alpha=2.00000000000000000000 \\
& f_{5}(x)=x^{3}+4 x^{2}-10, \alpha \approx 1.36523003414096845760 \\
& f_{6}(x)=e^{-x^{2}+x+2}-\cos (x+1)+x^{3}+1, \alpha=-1.000000000000000 \\
& f_{7}(x)=\sin ^{2}(x)-x^{2}+1, \alpha \approx 1.40449164821534122603 \\
& f_{8}(x)=\sqrt{x}-x, \alpha=1.00000000000000000
\end{aligned}
$$

The number of iteration (IT) for the compared methods are determined using the following stopping criteria $\left|x_{n+1}-x_{n}\right|<\varepsilon$, where $\varepsilon$ is the precision of the compared methods, while the COC is approximated by the formula 


$$
\rho \approx \frac{\ln \left|\left(x_{n+1}-\alpha\right) /\left(x_{n}-\alpha\right)\right|}{\ln \left|\left(x_{n}-\alpha\right) /\left(x_{n-1}-\alpha\right)\right|} .
$$

Table 1. The number of iteration for various iterative methods with $\varepsilon=10^{-95}$.

\begin{tabular}{lrrrccc}
\hline \multirow{2}{*}{$f(x)$} & $x_{0}$ & \multicolumn{5}{c}{ Number of iteration } \\
\cline { 3 - 7 } & & MN & MCK & MNS & MIOT & MMIOT \\
\hline \multirow{2}{*}{$f_{1}(x)$} & $-0,2$ & 8 & 5 & 5 & 5 & 4 \\
& 0,3 & 8 & 5 & 5 & 4 & 4 \\
\hline \multirow{2}{*}{$f_{2}(x)$} & 4,0 & 8 & 6 & 5 & 5 & 4 \\
& 4,5 & 7 & 5 & 5 & 4 & 4 \\
\hline \multirow{2}{*}{$f_{3}(x)$} & 0,1 & 8 & 5 & 5 & 5 & 4 \\
& 1,5 & 7 & 5 & 5 & 5 & 4 \\
\hline \multirow{2}{*}{$f_{4}(x)$} & 1,8 & 8 & 5 & 5 & 4 & 4 \\
\hline \multirow{2}{*}{$f_{5}(x)$} & 3,0 & 9 & 6 & 6 & 5 & 5 \\
\hline \multirow{2}{*}{$f_{6}(x)$} & 1,0 & 8 & 5 & 5 & 4 & 4 \\
\hline \multirow{2}{*}{$f_{7}(x)$} & 2,0 & 8 & 5 & 5 & 5 & 4 \\
\hline \multirow{2}{*}{$f_{8}(x)$} & 1,5 & 7 & 5 & 5 & 5 & 4 \\
& 0,0 & 7 & 6 & 5 & 6 & 4 \\
\hline
\end{tabular}

Besides using the Taylor expansion approach, we also use computational approximation using formulation in (33) to get the convergence order of the compared iterative method. The results as seen in Table 2. Table 2 describes that the computational order of convergence of the proposed method (MMIOT) is four. In addition to using the number of iterations and COCs, the performance of an iterative method can also be measured using accuracy, namely calculating the absolute of a function as given in Table 3. Table 3 shows the absolute value of the function using a total number of functional evaluations (TNFE), which are as many as twelve functional evaluations. Based on Table 3 , it can be seen that the proposed method has better accuracy than other iterative methods.

Table 2. The COC for the various iterative method with $\varepsilon=10^{-95}$

\begin{tabular}{ccccccc}
\hline \multirow{2}{*}{$f(x)$} & $x_{0}$ & \multicolumn{5}{c}{ COC } \\
\cline { 3 - 7 } & & MN & MCK & MNS & MIOT & MMIOT \\
\hline \multirow{2}{*}{$f_{1}(x)$} & $-0,2$ & 2,0000 & 2,9999 & 3,0000 & 3,0000 & 4,0000 \\
& 0,3 & 2,0000 & 3,0000 & 3,0000 & 3,0000 & 4,0000 \\
\hline \multirow{2}{*}{$f_{2}(x)$} & 4,0 & 2,0000 & 3,0000 & 3,0000 & 3,0000 & 4,0000 \\
& 4,5 & 2,0000 & 2,9999 & 3,0000 & 3,0000 & 4,0000 \\
\hline \multirow{2}{*}{$f_{3}(x)$} & 0,1 & 2,0000 & 3,0000 & 3,0000 & 3,0000 & 4,0000 \\
& 1,5 & 2,0000 & 2,9999 & 3,0000 & 3,0000 & 4,0000 \\
\hline \multirow{2}{*}{$f_{4}(x)$} & 1,8 & 2,0000 & 3,0000 & 3,0000 & 3,0000 & 4,0000 \\
& 3,0 & 2,0000 & 2,9999 & 3,0000 & 3,0000 & 4,0000 \\
\hline
\end{tabular}

Table 2. The COC for the various iterative method with $\varepsilon=10^{-95}$ (continued) 
New Modification of Behl's Method Free from Second Derivative with an Optimal Order of...

\begin{tabular}{ccccccc}
\hline \multirow{2}{*}{$f(x)$} & $x_{0}$ & \multicolumn{5}{c}{ COC } \\
\cline { 3 - 7 } & & MN & MCK & MNS & MIOT & MMIOT \\
\hline \multirow{2}{*}{$f_{5}(x)$} & 1,0 & 2,0000 & 3,0000 & 3,0000 & 3,0000 & 4,0000 \\
& 2,0 & 2,0000 & 2,9999 & 3,0000 & 3,0000 & 4,0000 \\
\hline \multirow{2}{*}{$f_{6}(x)$} & $-1,5$ & 2,0000 & 2,9999 & 3,0000 & 3,0000 & 4,0000 \\
& 0,0 & $2,0000)$ & 2,9999 & 3,0000 & 3,0000 & 4,0000 \\
\hline \multirow{2}{*}{$f_{7}(x)$} & 1,2 & 2,0000 & 3,0000 & 3,0000 & 3,0000 & 4,0000 \\
& 2,0 & 2,0000 & 2,9999 & 3,0000 & 3,0000 & 4,0000 \\
\hline \multirow{2}{*}{$f_{8}(x)$} & 0,5 & 2,0000 & 3,0000 & 3,0000 & 3,0000 & 4,0000 \\
& 1,5 & 2,0000 & 2,9999 & 3,0000 & 3,0000 & 4,0000 \\
\hline
\end{tabular}

Table 3. The absolute value of $f\left(x_{n}\right)$ for TNFE $=12$.

\begin{tabular}{rrrrrrr}
\hline \multirow{2}{*}{$f(x)$} & $x_{0}$ & \multicolumn{5}{c}{$\left|f\left(x_{n}\right)\right|$} \\
\cline { 3 - 7 } & & MN & MCK & MNS & \multicolumn{1}{c}{ MIOT } & MMIOT \\
\hline \multirow{2}{*}{$f_{1}(x)$} & $-0,2$ & $3,0850(\mathrm{e}-36)$ & $1,1432(\mathrm{e}-40)$ & $1,2725(\mathrm{e}-45)$ & $1,4315(\mathrm{e}-88)$ & $1,9110(\mathrm{e}-163)$ \\
& 0,3 & $1,0735(\mathrm{e}-42)$ & $1,7153(\mathrm{e}-43)$ & $9,0538(\mathrm{e}-54)$ & $1,5459(\mathrm{e}-101)$ & $1,0476(\mathrm{e}-193)$ \\
\hline \multirow{2}{*}{$f_{2}(x)$} & 4,0 & $5,0253(\mathrm{e}-33)$ & $1,8568(\mathrm{e}-29)$ & $5,5769(\mathrm{e}-42)$ & $6,4454(\mathrm{e}-81)$ & $3.5672(\mathrm{e}-158)$ \\
& 4,5 & $3,1919(\mathrm{e}-52)$ & $3,8771(\mathrm{e}-60)$ & $2,4261(\mathrm{e}-66)$ & $2,0154(\mathrm{e}-107)$ & $1,4627(\mathrm{e}-232)$ \\
\hline \multirow{2}{*}{$f_{3}(x)$} & 0,1 & $2,0345(\mathrm{e}-46)$ & $2,1012(\mathrm{e}-34)$ & $4,7468(\mathrm{e}-58)$ & $1,1084(\mathrm{e}-64)$ & $6,3477(\mathrm{e}-161)$ \\
& 1,5 & $3,7607(\mathrm{e}-64)$ & $9,4355(\mathrm{e}-50)$ & $3,5077(\mathrm{e}-80)$ & $6,2149(\mathrm{e}-53)$ & $4,9514(\mathrm{e}-202)$ \\
\hline \multirow{2}{*}{$f_{4}(x)$} & 1,8 & $2,8660(\mathrm{e}-41)$ & $1,3906(\mathrm{e}-39)$ & $4,2365(\mathrm{e}-52)$ & $5.2477(\mathrm{e}-98)$ & $6,5134(\mathrm{e}-181)$ \\
& 3,0 & $4,6449(\mathrm{e}-16)$ & $2,3970(\mathrm{e}-16)$ & $3,0961(\mathrm{e}-20)$ & $4,4874(\mathrm{e}-34)$ & $1,1038(\mathrm{e}-071)$ \\
\hline \multirow{2}{*}{$f_{5}(x)$} & 1,0 & $3,9823(\mathrm{e}-43)$ & $2,9959(\mathrm{e}-41)$ & $9,1052(\mathrm{e}-55)$ & $1,9038(\mathrm{e}-98)$ & $2,4510(\mathrm{e}-186)$ \\
& 2,0 & $1,2361(\mathrm{e}-37)$ & $6,1848(\mathrm{e}-41)$ & $7,8139(\mathrm{e}-48)$ & $1,9523(\mathrm{e}-65)$ & $3.6662(\mathrm{e}-162)$ \\
\hline \multirow{2}{*}{$f_{6}(x)$} & $-1,5$ & $5,7389(\mathrm{e}-66)$ & $7,4069(\mathrm{e}-51)$ & $5,1899(\mathrm{e}-92)$ & $1.9205(\mathrm{e}-40)$ & $1,3689(\mathrm{e}-167)$ \\
& 0,0 & $1,9261(\mathrm{e}-65)$ & $1,1267(\mathrm{e}-19)$ & $9,3636(\mathrm{e}-73)$ & $1.4610(\mathrm{e}-23)$ & $5,9424(\mathrm{e}-154)$ \\
\hline \multirow{2}{*}{$f_{7}(x)$} & 1,2 & $2,0864(\mathrm{e}-47)$ & $2,1333(\mathrm{e}-46)$ & $7,4954(\mathrm{e}-60)$ & $2,5292(\mathrm{e}-96)$ & $3,4470(\mathrm{e}-197)$ \\
& 2,0 & $2,2623(\mathrm{e}-32)$ & $3,9132(\mathrm{e}-32)$ & $8,2994(\mathrm{e}-41)$ & $2,8735(\mathrm{e}-45)$ & $8,9422(\mathrm{e}-126)$ \\
\hline \multirow{2}{*}{$f_{8}(x)$} & 0,5 & $1,5492(\mathrm{e}-43)$ & $1,2885(\mathrm{e}-12)$ & $2,2096(\mathrm{e}-55)$ & $1,8635(\mathrm{e}-54)$ & $1,1552(\mathrm{e}-130)$ \\
& 1,5 & $1,0649(\mathrm{e}-66)$ & $5,2351(\mathrm{e}-63)$ & $2,4094(\mathrm{e}-84)$ & $1,8528(\mathrm{e}-68)$ & $5,2043(\mathrm{e}-227)$ \\
\hline
\end{tabular}

\section{CONCLUSION}

We have developed a two-point iterative method with four orders convergence for $\beta=0$ to solve a nonlinear equation. The iterative method requires three evaluations of functions with an efficiency index equal to $4^{1 / 3} \approx 1.5874$. The numerical result which is given in Table 1,2 , and 3 show that the iterative method in equation (11) has better performance than other compared iterative methods. 


\section{REFERENCES}

[1] S. Chapra and R. Canale, Numerical Methods for Engineering, New York: Mc-Graw-Hill, Inc., 2010.

[2] J. Traub, Iterative Method do Solution of Equations, New York: Chelsea Publishing Company, 1964.

[3] A. Melman, "Geometry, and convergence of Euler's and Halley's methods," SLAM Review, vol. 39, no. 4, pp. 728-735, 1997.

[4] J. Sharma, "A family of third-order methods to solve nonlinear equations by quadratic curves approximation," Applied Mathematics, and Computation, vol. 184, pp. 210-215, 2007.

[5] S. Amat, S. Busquier and J. M. Gutierrez, "Geometric construction of iterative function to solve nonlinear equations," Journal of Computational and Applied Mathematics, vol. 157, pp. 197-205, 2003.

[6] S. Amat, S. Busquier, J. Guterrez and Hern, "On the global convergence of Chebyshev's method," Journal of Computational and Applied Mathematics, vol. 220, pp. 17-21, 2008.

[7] S. Abbasbandy, "Modified homotopy perturbation method for nonlinear equations and comparison with Adomian decomposition method," Applied Mathematics, and Computation, vol. 172, pp. 431-438, 2006.

[8] C. Chun, "Iterative methods improving Newton's method by the decomposition method," Computers and Mathematics with Applications, vol. 50, pp. 1559-1568, 2005.

[9] C. Chun and Y. Kim, "Several new third-order iterative methods for solving nonlinear equations," Acta Appl Math, vol. 109, pp. 1053-1063, 2010.

[10] F. Shah and M. Noor, "Variational iteration technique and some methods for the approximate solution of nonlinear equations," Applied Mathematics and Informatics Sciences Letter, vol. 2, no. 3, pp. 85-93, 2014.

[11] M. Baghat, "New two iterative methods for solving nonlinear equations," Journal of Mathematics Research, vol. 4, no. 3, p. 128-131, 2012.

[12] Wartono and T. Nanda, "Modifikasi metode Baghat tanpa turunan kedua dengan orde konvergensi optimal," in Prosiding Seminar Nasional Teknologi Informasi, Komunikasi dan Industri IX, Pekanbaru, 2017.

[13] H. Kung and J. Traub, "Optimal order of one-point and multipoint iteration," Journal of the Association for Computing Machinery, vol. 21, no. 4, p. 643-651, 1974.

[14] R. Behl, V. Kanwar and K. Sharma, "Another simple way of deriving several iterative functions to solve nonlinear equations," Journal of Applied Mathematics, 2012.

[15] J. Epperson, An Introduction to Numerical Methods and Analysis, New Jersey: John Willey \& Son, Inc, 2013.

[16] Wartono, M. Soleh, I. Suryani and Muhafzan, "Chebysev-Halley's Method without second derivative of eighth-order of convergence," Global Journal of Pure and Applied Mathematics, vol. 12, no. 4, pp. 2987-2997, 2016. 